\title{
Impedance Measurement of Wound Infection Status
}

\author{
A. Schröter ${ }^{1}$, A. Rösen-Wolff ${ }^{2}$, G. Gerlach $^{1}$ \\ ${ }^{1}$ Institute of Solid State Electronics, Technische Universität Dresden, 01062 Dresden, Germany \\ anna.schroeter@tu-dresden.de \\ ${ }^{2}$ Department of Pediatrics, University Hospital Carl Gustav Carus, 01307 Dresden, Germany
}

\begin{abstract}
:
Wound infection monitoring is a matter of high interest in wound treatment. Therefore, we extracted sensor parameters suitable to identify massive pathogen presence. One method is to measure neutrophil-derived extracellular DNA as an immediate response of the immune system. We proofed that impedance measurement is a reasonable measurement technique for this parameter. Our sensor concept comprises a plenary or fiber-based setup which could be integrated into a wound cover. Proof-of-principle experiments using impedance spectroscopy in cell cultures showed a dependency between extracellular DNA formation and impedance rise. This was verified by microscopic observation of fluorescently stained samples. A reproducible impedance signal deviation of in average $34 \%$ after lately 4 hours was observed.
\end{abstract}

Key words: health monitoring, wound infection, neutrophil extracellular traps, impedance spectroscopy, impedimetric sensors.

\section{Medical Background}

Recommended treatment of chronic wounds comes along with hydroactive wound covers containing hydrogels, hydrocolloids and foams. They extend the retention time of wound covers by several days which also enhances wound healing. This advantage is only sometimes used because of a possible infective complication. The wound cover is in most cases removed every 24 to 48 hours to check the infection status and to clean the wound. For this purpose the patient has to visit the clinic if he is treated ambulatory. However, these problems could be faced with an integrated, fast and mobile monitoring solution.

An infection goes by with the invasion of leukocytes, especially neutrophil granulocytes. From sensory perspective, granulocytes offer an interesting defense mechanism, the formation of neutrophil extracellular traps (NETs). They originate from the cell core which is dissolved when neutrophils sense a harmful microbe. Double-stranded (ds) DNA is released to the wound exudate. This reaction belongs to the inherent immune defense and is activated immediately after pathogen contact [1].

\section{Monitoring Concept}

From the clinical routine we can derive the most important requirements for a monitoring concept:
- an autonomous sensor solution

- capable to be integrated into a wound cover

- delivers fast and reliable infection information.

In former publications several wound monitoring concepts are introduced. They can be splitted roughly in optical [2] [3], gas-analytic [4], colorimetric and potentiometric or impedancebased methods [5] [6]. Considering the requirements the approaches by Vincenzini et al. [3] and Conolly et al. [5] seem to be promising, especially concerning integration potential. The former uses waveguide fibers integrated into a wound cover. A functional coating of hydrogel responds on parameters like $\mathrm{C}$-reactive protein and $\mathrm{pH}$ value. The coating changes its optical refraction index which is measured by a spectrometer. This optical measurement comes along with the need for brittle fibers which have the risk to break. Furthermore, the usage of mirrorgeometries and a spectrometer limit the capability for integration. An impedance-based concept as in [5] has not these disadvantages. The electrical measurement is performed with flexible conducting materials and can be miniaturized into a semiconductor designed chip. In this approach the parameter of interest is the amount of liquid and viscosity. Direct 
information to the infection status is not obtained.

Our investigations address directly the immune response of the body. With an impedancebased sensor we want to measure the NET formation and later other physiologically important parameter, for example temperature, $\mathrm{pH}$ value and elastase. This will be achieved by functional absorber materials like hydrogels. A possible planar setup is shown in Figure 1. A fiber-based solution which comes along with a higher flexibility in area coverage is also planned.

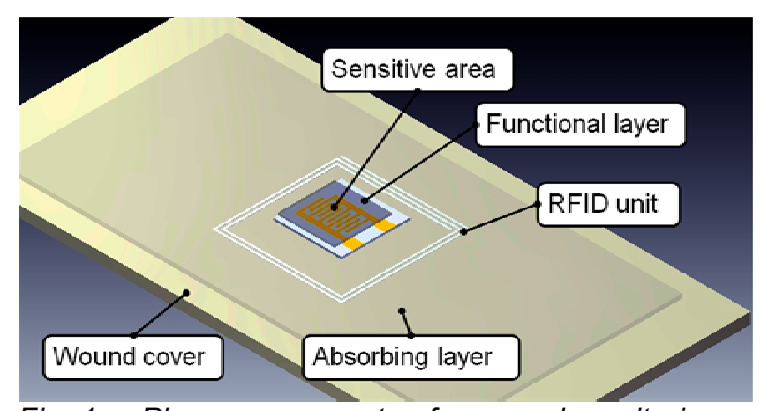

Fig. 1. Planar sensor setup for wound monitoring.

\section{Impedance Behavior of Cells and DNA}

Wound exudate consists of different constituents, which depend on the wound status: ionic, molecular and cell components. Healthy wound exudate has a similar composition as blood plasma and appears as a colorless fluid with low viscosity. The state of a wound infection is reached when a high amount of harmful pathogens accumulate to the wound site. Pathological exudate consists of a high number of leukocytes, which are fighting against an invasion of the agents and a spreading into the body. The NET formation is one of the fastest immune reactions. This negatively charged extracellular DNA is in high amounts present during an infection and leads to a change of the dielectric behavior of the exudate. The task of an impedance sensor is to measure this constituent. But the leukocytes itself and the varying ion strengths during the immune response affect the measurement, too.

Studying the dielectric behavior of dispersed DNA was an issue from the beginning of the 1980s [4] up to now, e.g. [5] [6] [7]. The described DNA-detection principles base on hybridization, labeling, specific binding and combinations of these in lab environments. Detection in the wound fluid comes along with more challenges. In contrary to purified DNA, the neutrophil extracellular DNA is more complex and is bound on proteins. The dielectric behavior of DNA still plays a role for an impedance detection of NETs. As the highly negatively charged DNA-backbone of NETs is mostly surrounded by a counter-ion shell a pure diffusive process during low-power electromagnetic excitation is excluded. More likely the movement of the counter-ions plays a role. The frequencies where these movements are in resonance with the excitation are called the cutoff frequencies. At these frequencies the dielectric losses pass through a maximum and the ability to store energy is lost with higher frequencies. Therefore, the real part of the permittivity drops. This phenomenon is named dispersion and occurs for the counter-ion shell in the low frequency range of some $\mathrm{Hz}$ [7] [8]. From 0.5 to $70 \mathrm{kHz}$ a second dispersion is described by Tomić et al. as a result of counter ion relaxations along a single DNA chain [9]. Hopping of counter ions between DNA chains of one DNA molecule causes a third smaller dispersion [8] [9]. It occurs in the range of $0.1 \mathrm{MHz}$ to $15 \mathrm{MHz}$ [9].

As NETs consist of decondensed elongated DNA only the interactions directly related to a single DNA chain are of interest, which affects the second dispersion mechanism. When the cell membrane of the neutrophil is intact it shields the electrical field especially in this frequency range. At this stage only the $\alpha$ - and $\beta$-dispersions of the cells can be measured. The $\alpha$-dispersion, from a few $\mathrm{mHz}$ to several $\mathrm{kHz}$ is caused by effects on the cell membrane and is more dominant if the cells have a negatively charged membrane [10]. For neutrophils this is not the case. The $\beta$ dispersion plays a more important role. This effect occurs in the middle frequency range up to several hundred $\mathrm{MHz}$ and results from capacitive effects on the cell membranes [11] [12]. In this range also the cell size is determinable as proposed by Schwan [12]. In cell suspensions often the $\gamma$-dispersion is also mentioned which lies in the range up to $100 \mathrm{GHz}$ and is caused by dipole mechanisms of water molecules and proteins. All ionic mechanisms appear in the low frequency range at some Hertz, where the slow diffusion properties of ions are dominant.

\section{Wound Model and Measurement}

To proof our sensor concept we used a cellbased wound model. Therefore neutrophil granulocytes are isolated from human blood samples by several centrifugation steps and erythrocyte lysis. The granulocytes are incubated in RPMI medium with autologous serum (2\%). For stimulation the chemical stimulant phorbol 12-myristate 13-acetate (PMA, 200 $\mu \mathrm{M}$ in the medium) is added. The cultivation is done with $5 \cdot 10^{4}$ or $2 \cdot 10^{6}$ cells in electrode-equipped wells. With a self-made 
adapter the electrodes are connected to an impedance analyzer which measures in the frequency range between $1 \mathrm{kHz}$ and $1 \mathrm{MHz}$. This setup with its single components is shown in Figure 2.

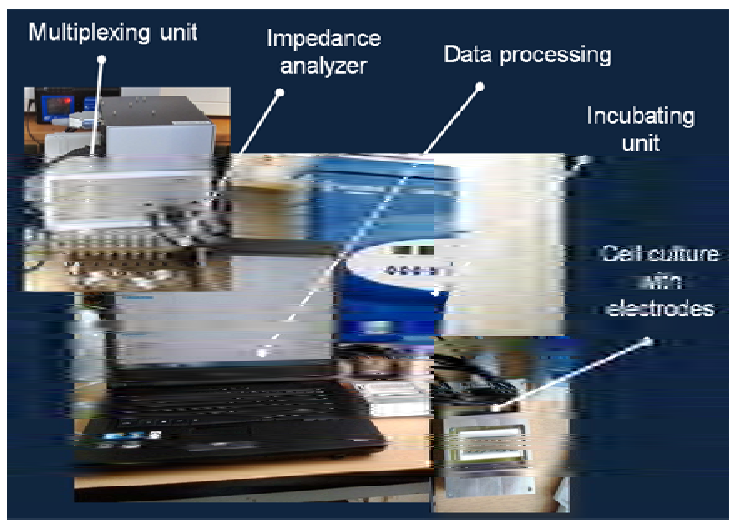

Fig. 2. Measurement setup for impedance characterization of extracellular DNA.

To confirm the NET formation with an alternative measurement we fluorescently stained the same samples measured with impedance spectroscopy. Fluorescent microscopy provides just a snapshot at a certain time point. Therefore, the reaction was stopped after a certain period of time with a fixation step with paraformaldehyde (4\%). The staining was done shortly before. Sytox Green $(5 \mu \mathrm{M})$ and DAPI $(300 \mathrm{nM})$ are used as ds DNAbinding dyes. Sytox Green does not intercalate and stains just extracellular DNA and the DNA of other dead cells. A demonstrative image detail is shown in Figure 3. Greyscaled images of the Sytox Green channel of a whole well were used for further processing. Image analysis comprises thresholding with the method of Otsu [13], automatic labeling of all objects and summing up the object areas. An absolute NET count is not available but the area of every well-picture is comparable to each other.

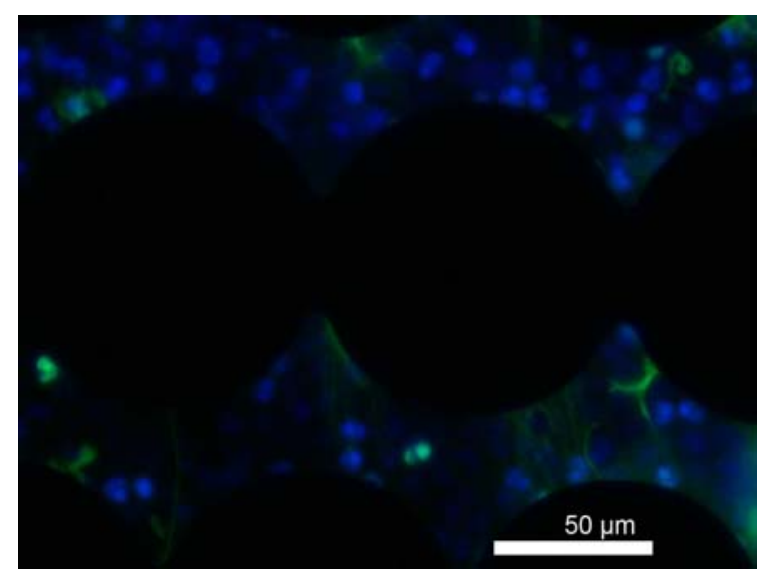

Fig. 3. Overlaid fluorescent image showing NET fibers and neutrophil cell cores (stained with Sytox
Green and DAPI respectively) on top of electrodes (black circles).

\section{NET-characterization with Impedance and Fluorescent Measurements}

Microscopic images of PMA-stimulated neutrophils show a formation of NET fibers as displayed in Figure 3. NET-forming cells have in the first stage a larger cell core area which is recognized by the threshold algorithm as displayed in Figure 4. Cells of apoptotic cells are filtered. Furthermore, the large NET areas are clearly extracted. Most of the cells settle on the edges. Therefore most NETs are recognized there.

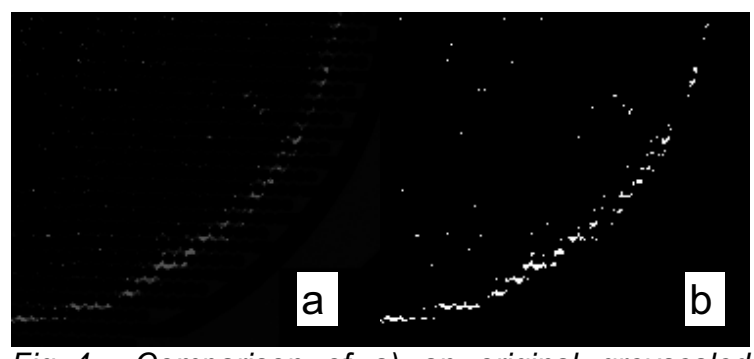

Fig. 4. Comparison of a) an original greyscaled image with b) a thresholded image.

A measurement of impedance with a following image analysis showed significant changes as shown in Figure 5. The impedance measurement starts shortly after stimulation. As only the changes are measured compared to the first value, the impedance change results in the value zero at time point zero. The fluorescent images show already a prestimulation. Possible reasons are the preparation conditions, which apply a stress to the neutrophils. Furthermore, unstimulated samples showed NETs which were in a much lower amount then the stimulated. The most deviations for the fluorescent area but also the impedance are at the maximum of about 2 to 3 hours.

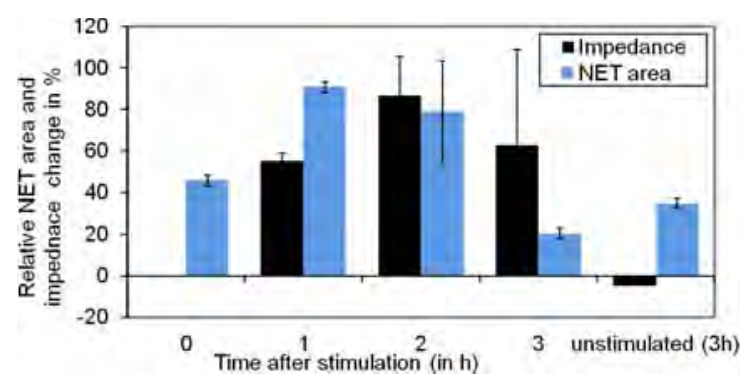

Fig. 5. NET area and corresponding impedance change (at $20 \mathrm{kHz}$ ), normalized at $99.3 \mathrm{Ohms}$ and 403,044 pixels respectively.

A statistical analysis of a high number of impedance measurements revealed the significance of the impedance deviations (Figure 6). But for a low cell number of $5 \cdot 10^{4}$ 
cells, where the well surface is rarely covered with cells the standard deviation is very high. For higher cell numbers stimulation was more certain. With the background that some cells are already pre-stimulated this result is explainable with the low amount of cells. Furthermore a cascade effect which is triggered by the release of transient reactive oxygen molecules can occur only limited due to the long intercellular paths. With $2 \cdot 10^{6}$ cells stimulation was more stable.

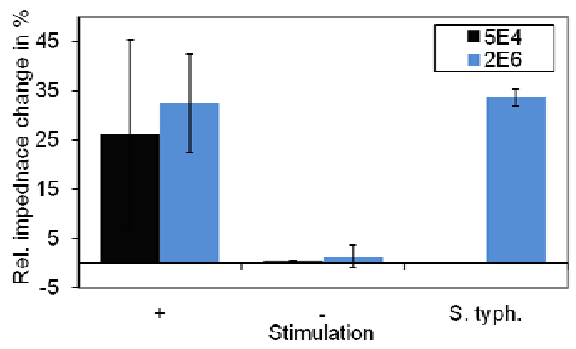

Fig. 6. Results of impedance measurements of PMA-stimulated (+), Salmonella typhimuriumstimulated (only $2 \cdot 10^{6}$ cells) and unstimulated neutrophils.

Table 1 summarizes the time to reach the maximum of impedance change for the same experiments. For $2 \cdot 10^{6}$ cells the maximum occurred in average after 3.6 hours. In the time behavior of some samples a second local maximum was observed at about 60 minutes. Until this point the rise is more rapid. The physical mechanism which lies behind these changes could be different, e.g. the different stages of the NET-formation. Furthermore, it is noticeable that the time point of the maximum is earlier for Salmonella-stimulated samples and for $5 \cdot 10^{4}$ neutrophils.

Tab. 1: Time to reach maximum of impedance change of PMA-stimulated and Salmonella typhimurium-stimulated (only $2 \cdot 10^{6}$ cells) with $2 \cdot 10^{6}$ and $5 \cdot 10^{4}$ neutrophils.

\begin{tabular}{|l|c|c|c|}
\hline Number of cells & $2 \cdot 10^{6}$ & $2 \cdot 10^{6}$ & $5 \cdot 10^{4}$ \\
\hline Stimulation & PMA & S. typh. & PMA \\
\hline Experiments & 21 & 4 & 12 \\
\hline Maximum time & $\mathbf{3 . 6}$ & $\mathbf{1 . 3}$ & $\mathbf{1 . 6}$ \\
\hline
\end{tabular}

\section{Summary}

NET formation as a possible recognition mechanism for wound infection status was examined. An impedance evaluation seems to be very promising concerning significance and reproducibility. The time behavior was studied in various experiments but still there are open questions left. For example the correlation between the physical mechanism that causes the impedance change is uncompleted. There are two approaches we are following to achieve this. On the one hand, we are overworking the fluorescent imaging evaluation. Especially a classification method for the different stages of NET formation is missing. On the other Hand, we want to do a dielectric modeling of the extracellular DNA. Of course, other important infection parameters - as mentioned above also have to be investigated. Together with one or two more reliable parameter the impedance change produced by NETs is a reliable measurand to monitor an infection in a wound cover.

\section{References}

[1] P. Martin, S. J. Leibovich, Inflammatory cells during wound repair: the good, the bad and the ugly, Trends in Cell Biology 15, 599-607 (2005); doi: 10.1016/j.tcb.2005.09.002

[2] D. G. Armstrong, L. A. Lavery, O. J. Liswood, F. W. Todd, J. A. Tredwell, Infrared Dermal Thermometry for the HighRisk Diabetic Foot, Physical Therapy 77, 2:169-175 (1997)

[3] P. Vincenzini, D. de Rossi, Wearable biosensors for monitoring wound healing. Advances in Science and Technology 57, 80-87 (2008); doi: 10.4028/www.scientific.net/AST.57.80

[4] A. L. P. S. Bailey, A. M. Pisanelli, K. C. Persaud, Development of conducting polymer sensor arrays for wound monitoring, Special Issue: Selected Papers from the 12th International Symposium on Olfaction and Electronic Noses - ISOEN 2007, International Symposium on Olfaction and Electronic Noses. Sensors and Actuators B: Chemical 131, 1:5-9 (2008); doi: 10.1016/j.snb.2007.12.035

[5] P. Conolly, D. McColl, Wound Dressings and Performance Measurement of Such Dressings, US020080171957A1 (18.04.2005)

[6] D. Sharp, J. Davis, Integrated urate sensors for detecting wound infection,

Electrochemistry Communications 10, 5:709-713 (2008); doi: 10.1016/j.elecom.2008.02.025

[7] M. Sakamoto, T. Fujikado, R. Hayakawa, Y. Wada, Low frequency dielectric relaxation and light scattering under ac electric field of DNA solutions, Biophysical Chemistry 11, 6:309-316 (1980); doi: 10.1016/03014622(80)87002-5

[8] S. Tomić, S. D. Babić, T.Vuletić, S. Krča, D. Ivanković, L. Griparić, R. Podgornik, Dielectric relaxation of DNA aqueous 
solutions, Physical Review E 75, 2:21905 (2007); doi: 10.1103/PhysRevE.75.021905

[9] S. Bone, C. A. Small, Dielectric studies of ion fluctuation and chain bending in native DNA, Biochimica et Biophysica Acta (BBA) Gene Structure and Expression 1260, 1:8593 (1995); doi: 10.1016/01674781(94)00181-2

[10] H. P. Schwan, Electrical properties of tissue and cell suspensions, Adv Biol Med Phys 5, 147-209 (1957)

[11] C. L. Davey, D. B. Kell, Low-frequency dielectric properties of biological cells, Bioelectrochemistry of Cells and Tissues, 159-207 (1995)

[12] H. P. Schwan, The Practical Success of Impedance Techniques from an Historical Perspective, Annals of the New York Academy of Sciences 873, 1:1-12 (1999); doi: 10.1111/j.1749-6632.1999.tb09443.x

[13] N. Otsu, A threshold selection method from gray level histograms, IEEE Trans. Syst. Man Cybern. SMC 9, 62-66 (1979) 